\title{
Universal Extensions of Restricted Classes of Quantum Operations
}

\author{
Michał Oszmaniec ${ }^{1,2}$ and Zoltán Zimborás ${ }^{3,4}$ \\ ${ }^{1}$ ICFO-Institut de Ciències Fotòniques, The Barcelona Institute of Science and Technology, \\ 08860 Castelldefels, Barcelona, Spain \\ ${ }^{2}$ Institute of Theoretical Physics and Astrophysics, National Quantum Information Centre, \\ Faculty of Mathematics, Physics and Informatics, University of Gdansk, Wita Stwosza 57, 80-308 Gdańsk, Poland \\ ${ }^{3}$ Wigner Research Centre for Physics, Hungarian Academy of Sciences, P.O. Box 49, H-1525 Budapest, Hungary \\ ${ }^{4}$ Dahlem Center for Complex Quantum Systems, Freie Universität Berlin, 14195 Berlin, Germany
}

(Received 26 May 2017; published 28 November 2017)

\begin{abstract}
For numerous applications of quantum theory it is desirable to be able to apply arbitrary unitary operations on a given quantum system. However, in particular situations only a subset of unitary operations is easily accessible. This raises the question of what additional unitary gates should be added to a given gate set in order to attain physical universality, i.e., to be able to perform arbitrary unitary transformation on the relevant Hilbert space. In this work, we study this problem for three paradigmatic cases of naturally occurring restricted gate sets: (A) particle-number preserving bosonic linear optics, (B) particle-number preserving fermionic linear optics, and (C) general (not necessarily particle-number preserving) fermionic linear optics. Using tools from group theory and control theory, we classify, in each of these scenarios, what sets of gates are generated, if an additional gate is added to the set of allowed transformations. This allows us to solve the universality problem completely for arbitrary number of particles and for arbitrary dimensions of the single-particle Hilbert space.
\end{abstract}

DOI: 10.1103/PhysRevLett.119.220502

In many applications of quantum mechanics it is important to have full control over a quantum system used to perform a desired task or a quantum protocol. This amounts to being able to implement arbitrary unitary operation on the system in question. Perhaps the most well-known example is the circuit model of quantum computing, where the ability to implement arbitrary unitary gates on a system of many distinguishable particles (say, qubits) is a necessary ingredient for performing universal quantum computation [1,2]. From the experimental perspective, it is typically very easy to implement single-qubit gates. This collection of gates, however, does not lead to universal quantum computation and to this aim has to be supplemented by an entangling gate [3]. Similar situations appear in other physical contexts. Typically, the set of easily accessible unitary gates acting on a given quantum system does not ensure full controllability.

This work studies the extension problem for gate sets appearing naturally in systems consisting of nondistinguishable particles: passive linear optics for $(A)$ bosonic and $(B)$ fermionic systems with fixed number of particles, as well as $(C)$ active linear optics acting on fermionic systems with fixed number of modes subject to the parity superselection rule [4]. Specifically, for the aforementioned scenarios, we study what unitary transformations on the relevant Hilbert space can be implemented if the restricted class of gates $K$ is supplemented by additional unitary transformations-see Fig. 1. We investigate two variants of this problem. (i) The gate set $K$ is supplemented with unitaries of the form $\exp (-i t X)$ generated by the
Hamiltonian $X$; (ii) the gate set $K$ is supplemented by a single unitary transformation $V$.

Linear optical transformations are relevant in many contexts. Passive bosonic linear optics describes singleparticle evolutions of a system of $N$ identical bosons in $d$ modes. Such transformations are natural for quantum optics, when quantum states of light pass through an optical network formed from beam splitters and phase shifters [5]. Linear optics underpins the Knill-Laflamme-Milburn (KLM) scheme of quantum computing with photons [6,7] and the boson sampling strategy for demonstrating quantum supremacy with linear optical networks [8]. Moreover, this class of transformations is used to manipulate cold bosonic particles in optical traps $[9,10]$. Similarly, passive fermionic linear optics describes single-particle evolutions of a system
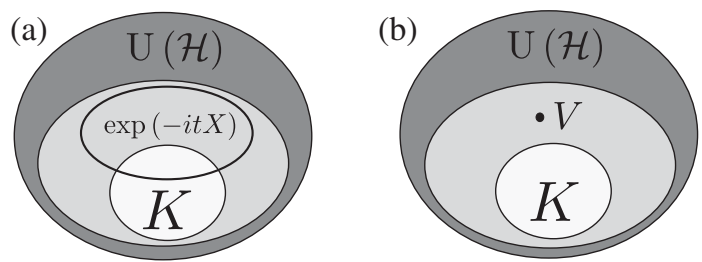

FIG. 1. A schematic presentation of the problems studied. (a) Given a family of gates $K$ (white) and a one-parameter family of unitaries $\exp (-i t X)$ (black loop), what class of gates (grey) can be generated in the full unitary group $U(\mathcal{H})$ (dark grey)? (b) Given a family of gates $K$ (white) and a single gate $V$ (black dot), what class of gates (grey) can be generated in the full unitary group $U(\mathcal{H})$ (dark grey)? 
of $N$ identical fermions in $d$ modes [11,12], which can, e.g., be realized in quantum-Hall-effect systems exhibiting edge channels [13]. Passive fermionic linear optics together with particle-number measurements yields a classically simulable model of quantum computation [12]. Moreover, this class of transformations has been recently used to study correlation [14] and nonlocality [15] properties in fermionic systems. Finally, active fermionic linear optics describes free-fermion transformations that are not necessarily particle-number preserving. These fermionic transformations are the basic ingredient of a classically simulable model of quantum computation $[4,11,12]$. This computational model has been even explored in the presence of noise [16-18], and can be connected, via the Jordan-Wigner transformation, to the model of computation based on Matchgates [19-22].

In this work, we completely solve problems (i) and (ii) for the scenarios $A-C$. We characterize the unitary transformations that are implementable (maybe approximately) by linear optical gates supplemented with any additional Hamiltonian or a gate. Our characterization is given in terms of explicit algebraic conditions on the Hamiltonian $X$ or the gate $V$ that can be tested operationally. The resulting behavior is surprisingly rich and structurally depends on the number of modes and the number of particles. In particular, contrary to what intuition might suggest, it is not true that every nontrivial extra gate or Hamiltonian provides universality in scenarios $A-C$. The solution of problems (i) and (ii) gives the clear understanding of what resources are necessary to have full physical controllability in the contexts listed above. Moreover, our results can be viewed as a step towards a solution of the general problem of classification of invertible quantum circuits posed recently by Aaronson and coworkers $[23,24]$. On the technical level we use techniques of the theory of Lie groups and Lie algebras, which have recently proved useful in studies on controllability of quantum systems [25-28] and on universality of gate sets [29-31].

Our general results have application to concrete physical examples. First, we consider the problem of extension to universality of passive bosonic linear optics for $d=2$ modes via cross-Kerr interaction [32], which is of relevance in quantum metrology with random bosonic states [33]. We also show that, quite surprisingly, there exist simple nonlinear Hamiltonians that do not lead to universality when added to passive fermionic or bosonic linear optics. Finally, it turns out that a simple quartic (in Majorana operators) Hamiltonian promotes active fermionic linear optics to universality in the positive-parity subspace.

Setting. - Let us start with formally defining the extension problem for (i) an additional Hamiltonian and (ii) an additional gate. In general we say that a unitary gate $U$ can be generated by unitaries from a set $\mathcal{S}$ if it can be approximated with arbitrary precision (in operator norm) by a sequence of products of gates from $\mathcal{S}$. We denote by $\langle K, X\rangle$ the set of unitaries that can be generated form the restricted gate set $K$ and unitaries of the form exp (-itX), where $t$ is an arbitrary real number. Likewise, slightly abusing the notation, we denote by $\langle K, V\rangle$ the set of unitaries that can be generated by elements from $K$ and an extra gate $V$. The aim of this work is to characterize sets $\langle K, X\rangle$ and $\langle K, V\rangle$ for different linear optical groups $K$ (acting on the appropriate Hilbert spaces $\mathcal{H}$ ). If the resulting gate set $\langle K, X\rangle$ (or $\langle K, V\rangle$ ) forms the full unitary group $U(\mathcal{H})$, we say that the Hamiltonian $X$ (or the gate $V$ ) promotes the restricted collection of gates $K$ to universality in $\mathcal{H}$. Since unitaries $U$ and $e^{i \alpha} U$ are physically indistinguishable, we assume, without loss of generality, that gates of the form $\exp (i \theta) \mathbb{1}$ are contained in $K$. In what follows by $\mathbb{T}(\mathcal{H})$ we denote the unitaries proportional to $\mathbb{1}$ on $\mathcal{H}$. We remark that the notion of physical universality in $\mathcal{H}$ introduced above does not imply computational universality in a sense of complexity theory [2].

We denote the Hilbert space of $N$ bosons in $d$ modes by $\mathcal{H}_{b}$. We have $\mathcal{H}_{b}=\operatorname{Sym}^{N}\left(\mathbb{C}^{d}\right)$; i.e., in this case the bosonic Hilbert space can be identified with the totally symmetric subspace of the Hilbert space of $N$ distinguishable qudits, $\left(\mathbb{C}^{d}\right)^{\otimes N}$. In this language the group of passive linear optical bosonic transformations, denoted by $\mathrm{LO}_{b}$, can defined as the group of unitaries of the form $U^{\otimes N}$, with $U \in U(d)$, restricted to the bosonic subspace $\mathcal{H}_{b}$.

The Hilbert space of $N$ (spinless) fermionic particles in $d$ modes $(d \geq N)$ is $\mathcal{H}_{f}=\wedge^{N}\left(\mathbb{C}^{d}\right)$, i.e., the totally antisymmetric subspace of $\left(\mathbb{C}^{d}\right)^{\otimes N}$. Similarly to the bosonic case, the group of passive fermionic linear optics $\mathrm{LO}_{f}$ is defined as the group of unitaries $U^{\otimes N}$, with $U \in U(d)$, restricted to the fermionic subspace $\mathcal{H}_{f}$.

In the case of the fermionic system without the restricted number of particles, the relevant Hilbert space is the direct sum of the different $N$-particle fermionic Hilbert spaces, i.e., the Fock space $\mathcal{H}_{\text {Fock }}=\oplus_{N=0}^{d} \wedge^{N} \mathbb{C}^{d}$. $\mathcal{H}_{\text {Fock }}$ is spanned by the Fock states $\left|n_{1}, \ldots, n_{d}\right\rangle$, with $n_{k} \in\{0,1\}$. In this space we have fermionic creation and annihilation operators, $f_{k}^{\dagger}$, $f_{k}$ satisfying the canonical anticommutation relations and one can define the standard number operators $\hat{n}_{k}=f_{k}^{\dagger} f_{k}$. It is also convenient to introduce Majorana fermion operators $m_{2 k-1}=f_{k}+f_{k}^{\dagger}, \quad m_{2 k}=i\left(f_{k}-f_{k}^{\dagger}\right), \quad$ for $\quad k=1, \ldots, d$. Majorana operators satisfy the anticommutation relations $\left\{m_{i}, m_{j}\right\}=2 \delta_{i j} \mathbb{1}$. In many situations fermionic systems obey the so-called parity superselection rule [4,27,34], which states that any physically accessible operations must commute with the total parity operator $Q=(-1)^{\sum_{k=1}^{d} \hat{n}_{k}}$. In this work, we restrict our attention to the positive-parity subspace $\mathcal{H}_{\text {Fock }}^{+}$, i.e., the subspace spanned by Fock states with an even number of particles. Results completely analogous to the ones presented here can be obtained also for the negative-parity subspace. Active fermionic linear optics acting in $\mathcal{H}_{\text {Fock }}$ consists of unitaries of the form $\exp \left(\sum_{l, k=1}^{2 d} h_{k l} m_{k} m_{l}\right)$, where $h_{k l}$ is a real antisymmetric 
$2 d \times 2 d$ matrix. Since we are interested only in the positiveparity subspace $\mathcal{H}_{\text {Fock}}^{+}$, we formally define the group of active fermionic linear transformations as the group consisting of unitaries $[\exp (i \phi) \mathbb{1}] \exp \left(\sum_{l, k=1}^{2 d} h_{k l} m_{k} m_{l}\right)$, restricted to $\mathcal{H}_{\text {Fock}}^{+}$.

The groups introduced above are compact Lie subgroups of the unitary group $U(\mathcal{H})$, where $\mathcal{H}$ is the Hilbert space describing the relevant physical system. For a given Lie group $K \subset U(\mathcal{H})$, it is convenient to work with the corresponding Lie algebra consisting of Hamiltonians that generate (via exponentiation) the unitaries belonging to $K$. The Lie algebras of the groups studied in this paper turn out to be (up to the trivial generators proportional to identity) simple Lie algebras [35] represented irreducibly on the relevant Hilbert spaces. This observation is crucial for obtaining our central results given in theorems 1-4. In particular, this allows us to use the classification results by Dynkin [36] concerning the maximal Lie subalgebras of simple Lie algebras (see also [26] for an illustration of these results in a physical context, and [37] for a detailed mathematical exposition).

For the sake of clarity of the presentation, we moved technical proofs to Supplemental Material [38] and focused on the discussion of the physical meaning of our results.

Application.-Before stating our results in full generality, let us present first an exemplary application of our findings. In Ref. [33], the authors were interested in extending bosonic linear optics to universality in $\mathcal{H}_{b}$ by adding an additional gate. This problem was motivated by the need to construct a physically accessible universal gate set in $\mathcal{H}_{b}$, which can be used to generate, via construction based on random circuits [45], approximate bosonic $t$-designs. The example below proves that a single gate based on the cross-Kerr nonlinearity suffices to promote bosonic linear optics to universality in $\mathcal{H}_{b}$. It should be mentioned that Kerr-like transformation have been previously used to obtain universal quantum computation in continuous-variable systems [46].

Example 1. Consider a bosonic system with $d=2$ modes and $N>1$ particles, and a gate generated by the cross-Kerr interaction (acting on $\mathcal{H}_{b}$ for a fixed time $t$ ), $V_{t}=\exp \left(-i t \hat{n}_{a} \hat{n}_{b}\right)$, where $\hat{n}_{a, b}$ are the occupation-number operators corresponding to modes $a$ and $b$. Let $\left\langle\mathrm{LO}_{b}, V_{t}\right\rangle$ be the group of transformations generated by passive bosonic linear optics and $V_{t}$. Then, $\left\langle\mathrm{LO}_{b}, V_{t}\right\rangle=U\left(\mathcal{H}_{b}\right)$ if and only if $e^{2 i t[l(N-l)-k(N-k)]} \neq 1$, for at least one pair $(k, l)$, where $k, l=0, \ldots, N$. In particular, the gate $V_{(\pi / 3)}$ promotes passive bosonic linear optics to universality in $\mathcal{H}_{b}$ for $d=2$ modes.

The above result follows directly from theorem 1 stated below (see Supplemental Material [38] for the explicit computation).

Main results.-We start with the presentation of results concerning passive bosonic linear optics.

Theorem 1 [Extensions of passive bosonic linear optics with an additional gate] Let $V \notin \mathrm{LO}_{b}$ be a gate acting on the
Hilbert space $\mathcal{H}_{b}$ of $N>1$ bosons in $d$ modes. Let $\left\langle\mathrm{LO}_{b}, V\right\rangle$ be the group of transformations generated by passive bosonic linear optics and $V$ in $\mathcal{H}_{b}$. For $d=2$ we define

$\mathbb{L}_{b}=\left|\Psi_{b}\right\rangle\left\langle\Psi_{b}|,| \Psi_{b}\right\rangle=\sum_{k=0}^{N}(-1)^{k}\left|D_{k}\right\rangle\left|D_{N-k}\right\rangle \in \mathcal{H}_{b} \otimes \mathcal{H}_{b}$,

where $\left|D_{k}\right\rangle$ denote the two-mode Dicke states with $k$ particles being in the first mode. We have the following possibilities: (a) If $d>2$, then $\left\langle\mathrm{LO}_{b}, V\right\rangle=U\left(\mathcal{H}_{b}\right)$. (b) If $d=2, N \neq 6$ and $\left[V \otimes V, \mathbb{L}_{b}\right]=0$, then

$$
\left\langle\mathrm{LO}_{b}, V\right\rangle=G_{b}=\left\{U \in U\left(\mathcal{H}_{b}\right) \mid\left[U \otimes U, \mathbb{L}_{b}\right]=0\right\} .
$$

(c) If $d=2$ and $\left[V \otimes V, \mathbb{L}_{b}\right] \neq 0$, then $\left\langle\mathrm{LO}_{b}, V\right\rangle=U\left(\mathcal{H}_{b}\right)$. In the above theorem we have situations with $N=1$ particles as for them $\mathrm{LO}_{b}=U\left(\mathcal{H}_{b}\right)$. We see that for $d \neq 2$ any additional gate promotes $\mathrm{LO}_{b}$ to universality in the bosonic space $\mathcal{H}_{b}$. For $d=2$ the resulting gate set $\left\langle\mathrm{LO}_{b}, V\right\rangle$ depends only on the commutator $\left[V \otimes V, \mathbb{L}_{b}\right]$. If it is nonzero, then $V$ again extends $\mathrm{LO}_{b}$ to universality; while if it vanishes (and $N \neq 6$ ) $V$ extends $\mathrm{LO}_{b}$ to the "middle group" $G_{b}$. Up to a global phase the group $G_{b}$ consists of unitaries that preserve the bilinear form defined by $B(|\psi\rangle,|\phi\rangle)=\left\langle\Psi_{b}|(|\psi\rangle \otimes|\phi\rangle)\right.$. Here, by preservation we mean that $B_{b}(U|\psi\rangle, U|\phi\rangle)=B_{b}(|\psi\rangle,|\phi\rangle)$, for all vectors $|\phi\rangle,|\psi\rangle$. If the number of particles $N$ is even then $\left|\Psi_{b}\right\rangle$ is a symmetric tensor and defines the real inner product. In this case we have $G_{b}=\left\langle\mathbb{T}\left(\mathcal{H}_{b}\right), \operatorname{SO}\left(\mathcal{H}_{b}\right)\right\rangle$, where $S O\left(\mathcal{H}_{b}\right)$ is the special orthogonal group on $\mathcal{H}_{b}$. When the number of particles $N$ is odd, the vector $\left|\Psi_{b}\right\rangle$ is antisymmetric and defines the symplectic structure (i.e., nondegenerate and antisymmetric form) on $\mathcal{H}_{b}$. In this case we have $G_{b}=\left\langle\mathbb{T}\left(\mathcal{H}_{b}\right), \operatorname{USp}\left(\mathcal{H}_{b}\right)\right\rangle$, where $\operatorname{USp}\left(\mathcal{H}_{b}\right)$ is the unitary symplectic group. The two differ considerably as $\operatorname{USp}(\mathcal{H})$ acts transitively on the set of pure states on $\mathcal{H}$ $[27,47,48]$. On the other hand, $\mathrm{SO}(\mathcal{H})$ acts transitively only on real pure states. Thus, for an odd number of particles, adding any additional gate gives either the full unitary controllability or the pure-state controllability. In the case of $d=2$ modes and $N=6$ particles if $\left[V \otimes V, \mathbb{L}_{b}\right]=0$ the situation complicates due to the presence of an additional group (related to the exotic group $G_{2}$ ) in between $\mathrm{LO}_{b}$ and $G_{b}$. We leave the description of this exceptional case as an interesting open problem.

From theorem 1 one can infer the following result concerning the additional Hamiltonian $X$ acting on $\mathcal{H}_{b}$ [intuitively, one can obtain it by setting $V=\exp (i t X)$ in theorem 1 and differentiating over $t$ ].

Theorem 2 [Extensions of passive bosonic linear optics via an additional Hamiltonian] Let $X \notin \operatorname{Lie}\left(\mathrm{LO}_{b}\right)$ be a Hamiltonian acting on Hilbert space of $N$ bosons in $d$ modes $\mathcal{H}_{b}$. Let $\left\langle\mathrm{LO}_{b}, X\right\rangle$ be the group of transformations generated by passive bosonic linear optical optics and 
$X$ in $\mathcal{H}_{b}$. We have the following possibilities. (a) If $d>2$, then $\left\langle\mathrm{LO}_{b}, X\right\rangle=U\left(\mathcal{H}_{b}\right)$. (b) If $d=2, \quad N \neq 6$, and $\left[X \otimes \mathbb{1}+\mathbb{1} \otimes X, \mathbb{L}_{b}\right]=0$, then

$\left\langle\mathrm{LO}_{b}, X\right\rangle=G_{b}=\left\{U \in U\left(\mathcal{H}_{b}\right) \mid\left[U \otimes U, \mathbb{L}_{b}\right]=0\right\}$.

(c) If $d=2$ and $\left[X \otimes \mathbb{1}+\mathbb{1} \otimes X, \mathbb{L}_{b}\right] \neq 0$, then $\left\langle\mathrm{LO}_{b}, X\right\rangle=U\left(\mathcal{H}_{b}\right)$.

Combining the above result and the discussion below theorem 1 we see that there might exist physical Hamiltonians that add different controllability properties to $\mathrm{LO}_{b}$, depending on the number of particles $N$. The following example shows that this is indeed the case.

Example 2. Consider the Hamiltonian $X_{3}=\hat{n}_{a}^{3}-\hat{n}_{b}^{3}$ acting on $\mathcal{H}_{b}$ for $d=2$ modes and $N \neq 6$ particles. Deepening on the value $N$ we get different types of gate sets after supplementing passive bosonic optics with $X_{3}$. (a) For even $N,\left\langle\mathrm{LO}_{b}, X_{3}\right\rangle=\left\langle\mathbb{T}\left(\mathcal{H}_{b}\right), \mathrm{SO}\left(\mathcal{H}_{b}\right)\right\rangle$. (b) For odd $N,\left\langle\mathrm{LO}_{b}, X_{3}\right\rangle=\left\langle\mathbb{T}\left(\mathcal{H}_{b}\right), \mathrm{USp}\left(\mathcal{H}_{b}\right)\right\rangle$. In particular, for odd $N$ we have full controllability on the set of pure states on $\mathcal{H}_{b}$, whereas for even $N$ this is not the case.

We now move to the discussion of fermionic linear optics (both passive and active). In the main text we present the results concertinaing the gate extension problem (ii). The corresponding theorems for the Hamiltonian extension problem are given in Supplemental Material [38] and their relation with the results presented in the main text is analogous to the connection between theorem 2 and theorem 1.

Theorem 3 [Extensions of passive fermionic linear optics with an additional gate] Let $V \notin \mathrm{LO}_{f}$ be a gate acting on Hilbert space of $N$ fermions in $d$ modes $\mathcal{H}_{f}$, where $N \notin\{0,1, d-1, d\}$. Let $\left\langle\mathrm{LO}_{f}, V\right\rangle$ be the group of transformations generated by passive fermionic linear optics and $V$ in $\mathcal{H}_{f}$. For $d=2 N$ (half-filling) we define

$$
\begin{aligned}
\mathbb{L}_{f} & =\left|\Psi_{f}\right\rangle\left\langle\Psi_{f}|, \operatorname{with}| \Psi_{f}\right\rangle \\
& =|1\rangle \wedge|2\rangle \wedge \ldots \wedge|2 N\rangle \in \mathcal{H}_{f} \otimes \mathcal{H}_{f}
\end{aligned}
$$

where $\wedge$ denotes the standard wedge product. We have the following possibilities: (a) If $d \neq 2 N$, then $\left\langle\mathrm{LO}_{f}, V\right\rangle=$ $U\left(\mathcal{H}_{f}\right)$. (b) If $d=2 N$ and $V=W k$, for $k \in \mathrm{LO}_{f}$ and $W=\prod_{i=1}^{d}\left(f_{i}+f_{i}^{\dagger}\right)$, then $\left\langle\mathrm{LO}_{f}, V\right\rangle=\mathrm{LO}_{f} \cup \mathrm{LO}_{f} W$. (c) If $d=2 N, V \neq g W$, for $g \in \mathrm{LO}_{f}$, and $\left[V \otimes V, \mathbb{L}_{f}\right]=0$, then

$$
\left\langle\mathrm{LO}_{f}, V\right\rangle=G_{f}=\left\{U \in U\left(\mathcal{H}_{f}\right) \mid\left[V \otimes V, \mathbb{L}_{f}\right]=0\right\} .
$$

(d) If $d=2 N$ and $\left[V \otimes V, \mathbb{L}_{f}\right] \neq 0$, then $\left\langle\mathrm{LO}_{f}, V\right\rangle=$ $U\left(\mathcal{H}_{f}\right)$.

The structure of the above result is similar to the case of passive bosonic linear optics. In the formulation of the theorem we have excluded the noninteresting cases $N \in\{0,1, d-1, d\}$ since for them $\mathrm{LO}_{f}$ equals the full unitary group on the respective Hilbert space. When $d \neq 2 N$ every gate promotes $\mathrm{LO}_{f}$ to universality. However, in the physically relevant case of half-filling [49], a more interesting "onion" structure appears. In the case (b) addition of an extra gate of the form $k W$, where $k \in \mathrm{LO}_{f}$ and gate $W$ (describing particle-hole transformation in $\mathcal{H}_{f}$ ) gives the gate set $\mathrm{LO}_{f} \cup \mathrm{LO}_{f} W$ (it is crucial here that $W$ commutes with $\mathbb{L}_{f}$ and that conjugation by $W$ leaves $\mathrm{LO}_{f}$ invariant). The further possibilities are described, similarly to the bosonic case, by the commutation properties of $V \otimes V$ with $\mathrm{LO}_{f}$. If $d$ is not divisible by 4 we have $G_{f}=\left\langle\mathbb{T}\left(\mathcal{H}_{f}\right), \operatorname{SO}\left(\mathcal{H}_{f}\right)\right\rangle$. On the other hand, for $d$ divisible by $4, G_{f}=\left\langle\mathbb{T}\left(\mathcal{H}_{f}\right), \operatorname{USp}\left(\mathcal{H}_{f}\right)\right\rangle$. The corresponding bilinear forms are defined by inner products with $\left|\Psi_{f}\right\rangle$. Using theorem 3, one can efficiently obtain results on extensions to universality, as the one given by the next example.

Example 3. For any nonquadratic Hamiltonian $M$ containing only two-mode terms, the generated group $\left\langle\mathrm{LO}_{f}, M\right\rangle$ is the entire unitary group $U\left(\mathcal{H}_{f}\right)$.

Hamiltonians that are not composed of two-mode terms are also often studied. One typical family of these are the so-called correlated hopping Hamiltonians, where the hopping term between two sites is multiplied with number operators belonging to other sites. For such Hamiltonians universality is not guaranteed.

Example 4. Consider the correlated hopping Hamiltonian

$$
\begin{aligned}
Y= & \sum_{j=1}^{d / 2-1}\left(\hat{n}_{2 j}-\hat{n}_{2 j+2}\right)^{2}\left(f_{2 j-1}^{\dagger} f_{2 j+1}+f_{2 j+1}^{\dagger} f_{2 j-1}\right) \\
& +\left(\hat{n}_{2 j-1}-\hat{n}_{2 j+1}\right)^{2}\left(f_{2 j}^{\dagger} f_{2 j+2}+f_{2 j+2}^{\dagger} f_{2 j}\right),
\end{aligned}
$$

acting on $\mathcal{H}_{f}$ for the case of half-filling $(d=2 N)$. Then, we have the following situations: (a) for even $N,\left\langle\mathrm{LO}_{f}, Y\right\rangle=$ $\left\langle\mathbb{T}\left(\mathcal{H}_{f}\right), \mathrm{SO}\left(\mathcal{H}_{f}\right)\right\rangle$; (b) for odd $N,\left\langle\mathrm{LO}_{f}, Y\right\rangle=\left\langle\mathbb{T}\left(\mathcal{H}_{f}\right)\right.$, $\left.\operatorname{USp}\left(\mathcal{H}_{f}\right)\right\rangle$. For odd $N$ the Hamiltonian $Y$ together with $\mathrm{LO}_{f}$ ensures full controllability on the set of pure states on $\mathcal{H}_{f}$. However, for even $N$ this is not the case. The above statements are even true for each term appearing in sum Eq. (4). The correlated hopping Hamiltonian $Y$ often appears (in a relabeled form) in the literature on extended Hubbard models [50].

Our last result concerns the extension problem for active fermionic linear optics.

Theorem 4 [Extensions of active fermionic linear optics via additional gate] Let $\langle$ FLO, $V\rangle$ be the group of transformations generated by active linear optics and $V$ acting in positive-parity Fock subspace $\mathcal{H}_{\text {Fock }}^{+}$with $d>3$ modes. For $d=2 k$ we define

$$
\mathbb{L}_{\mathrm{FLO}}=\frac{1}{2^{d(2 d-1)}} \prod_{1 \leq i<j \leq 2 d}\left(I \otimes I+m_{i} m_{j} \otimes m_{i} m_{j}\right) .
$$


We have the following possibilities: (a) If $d \neq 2 k$, then $\langle$ FLO, $V\rangle=U\left(\mathcal{H}_{\text {Fock }}^{+}\right) \quad$ (b) If $d=2 k$, and $\left[V \otimes V, \mathbb{L}_{\mathrm{FLO}}\right]=0$, then

$\langle\mathrm{FLO}, V\rangle=G_{\mathrm{FLO}}=\left\{U \in U\left(\mathcal{H}_{\mathrm{Fock}}^{+}\right) \mid\left[U \otimes U, \mathbb{L}_{\mathrm{FLO}}\right]=0\right\}$.

(c) If $d=2 k$ and $\left[V \otimes V, \mathbb{L}_{\mathrm{FLO}}\right] \neq 0$, then $\langle\mathrm{FLO}, V\rangle=$ $U\left(\mathcal{H}_{\text {Fock }}^{+}\right)$.

In the above result be have omitted cases $d \leq 3$ as for them FLO is itself the full unitary group on $\mathcal{H}_{\text {Fock }}^{+}$[17]. If the number of modes is not even, then every gate promotes FLO to universality in $\mathcal{H}_{\mathrm{Fock}}^{+}$. If the number of modes is even, then an additional gate either (c) promotes FLO to universality or extends it to the group $G_{\mathrm{FLO}}$ (b). Furthermore, analogously to the case of passive fermionic optics, if $d$ is divisible by $4 G_{\text {FLO }}=\left\langle\mathbb{T}\left(\mathcal{H}_{\text {Fock }}^{+}\right), \operatorname{SO}\left(\mathcal{H}_{\text {Fock }}^{+}\right)\right\rangle$ and if this is not the case $G_{\text {FLO }}=\left\langle\mathbb{T}\left(\mathcal{H}_{\text {Fock }}^{+}\right), \mathrm{USp}\left(\mathcal{H}_{\text {Fock }}^{+}\right)\right\rangle$ (a detailed description of the corresponding orthogonal and symplectic forms is given in Supplemental Material [38]).

Example 5. For arbitrary number of modes $d>3$ the interaction $H_{\text {in }}=m_{1} m_{2} m_{3} m_{3}$ extends FLO to universality in $\mathcal{H}_{\text {Fock }}^{+}$, i.e., $\left\langle\right.$FLO,$\left.H_{\text {in }}\right\rangle=U\left(\mathcal{H}_{\text {Fock }}^{+}\right)$. This result suggests that time-independent Hamiltonian $\mathcal{H}_{\text {in }}$ together with linear optics allows us to perform efficient quantum computation (if the standard occupation-number measurements are allowed) [51,52] .

Discussion.-In this Letter, we presented a comprehensive treatment of the extension problems for various classes of linear optical gates for bosons and fermions. The resulting behavior is surprisingly rich and critically depends on the number of modes and number of particles present in the system. However, there are a number of interesting problems we did not address here. First, it would be interesting to analyze which extra gates or Hamiltonians allow for the most efficient control [53] or the efficient approximation of gates from the appropriate unitary group [54]. Another important problem concerns the robustness of the extra gate or Hamiltonian to the noise that inevitably affects any quantum system. In future works we also plan to use our results to study (computational) universality of classically simulable models of computation supported on fermionic systems [11] and Machgates [19-22].

We thank Antonio Acín, Jens Eisert, Christian Gogolin, Adam Sawicki, and Robert Zeier for interesting and stimulating discussions. This work has been supported by the European Research Council (Consolidator Grant No. 683107/CoG QITBOX), Spanish Ministry of Economy and Competitiveness (Grant No. QIBEQI FIS2016-80773$P$, and Severo Ochoa Grant No. SEV-2015-0522), Fundaci'o Privada Cellex, Generalitat de Catalunya (Grant No. SGR 874, 875, and CERCA Programme). M. O. acknowledges the support of the Homing program of the Foundation for Polish Science cofinanced by the European Union under the European Regional
Development Fund. We also acknowledge the support of the Hungarian National Research, Development and Innovation Office (Grants No. NKFI K 124152, No. K 124351, No. K 124176), and Janos Bolyai Scholarship of the Hungarian Academy of Sciences.

[1] A. Y. Kitaev, A. Shen, and M. N. Vyalyi, Classical and Quantum Computation (American Mathematical Society, Providence, 2002), Vol. 47.

[2] M. A. Nielsen and I. L. Chuang, Quantum Computation and Quantum Information (Cambridge University Press, Cambridge, 2010).

[3] J.-L. Brylinski and R. Brylinski, Universal quantum gates, in Mathematics of Quantum Computation, edited by R. Brylinski and G. Chen (Chapman \& Hall/CRC Press, Boca Raton, Florida, 2002).

[4] S. B. Bravyi and A. Y. Kitaev, Ann. Phys. (San Diego) 298, 210 (2002).

[5] J. Carolan, C. Harrold, C. Sparrow, E. Martín-López, N. J. Russell, J. W. Silverstone, P. J. Shadbolt, N. Matsuda, M. Oguma, M. Itoh et al., Science 349, 711 (2015).

[6] E. Knill, R. Laflamme, and G. J. Milburn, Nature (London) 409, 46 (2001).

[7] P. Kok, W. J. Munro, K. Nemoto, T. C. Ralph, J. P. Dowling, and G. J. Milburn, Rev. Mod. Phys. 79, 135 (2007).

[8] S. Aaronson and A. Arkhipov, in Proceedings of the FortyThird Annual ACM Symposium on Theory of Computing (ACM, New York, 2011), pp. 333-342.

[9] G. J. Milburn, J. Corney, E. M. Wright, and D. F. Walls, Phys. Rev. A 55, 4318 (1997).

[10] M. Albiez, R. Gati, J. Fölling, S. Hunsmann, M. Cristiani, and M. K. Oberthaler, Phys. Rev. Lett. 95, 010402 (2005).

[11] B. M. Terhal and D. P. DiVincenzo, Phys. Rev. A 65, 032325 (2002).

[12] D. P. DiVincenzo and B. M. Terhal, Found. Phys. 35, 1967 (2005).

[13] E. Bocquillon, V. Freulon, F. D. Parmentier, J.-M. Berroir, B. Plaçais, C. Wahl, J. Rech, T. Jonckheere, T. Martin, C. Grenier et al., Ann. Phys. (Berlin) 526, 1 (2014).

[14] M. C. Tichy, J. Phys. B 47, 103001 (2014).

[15] D. Dasenbrook, J. Bowles, J. B. Brask, P. P. Hofer, C. Flindt, and N. Brunner, New J. Phys. 18, 043036 (2016).

[16] S. Bravyi and R. Koenig, Quantum Inf. Comput. 12, 925 (2012).

[17] F. de Melo, P. Ćwikliński, and B. M. Terhal, New J. Phys. 15, 013015 (2013).

[18] M. Oszmaniec, J. Gutt, and M. Kuś, Phys. Rev. A 90, 020302 (2014)

[19] L. G. Valiant, SIAM J. Comput. 31, 1229 (2002).

[20] E. Knill, arXiv:0108033.

[21] R. Jozsa and A. Miyake, in Proceedings of the Royal Society of London A, Mathematical, Physical and Engineering Sciences (The Royal Society, London, 2008), pp. 30893106.

[22] D. J. Brod, Phys. Rev. A 93, 062332 (2016).

[23] A. Bouland and S. Aaronson, Phys. Rev. A 89, 062316 (2014).

[24] S. Aaronson, D. Grier, and L. Schaeffer, arXiv:1504.05155. 
[25] C. Altafini, J. Math. Phys. 43, 2051 (2002).

[26] R. Zeier and T. Schulte-Herbrüggen, J. Math. Phys. 52, 113510 (2011).

[27] Z. Zimborás, R. Zeier, M. Keyl, and T. Schulte-Herbrüggen, EPJ Quantum Techno. 1, 11 (2014).

[28] Z. Zimborás, R. Zeier, T. Schulte-Herbrüggen, and D. Burgarth, Phys. Rev. A 92, 042309 (2015).

[29] A. Sawicki, Quantum Inf. Comput. 16, 0291 (2016).

[30] A. Sawicki and K. Karnas, Ann. Henri Poincare 18, 3515 (2017).

[31] A. Sawicki and K. Karnas, Phys. Rev. A 95, 062303 (2017).

[32] D. J. Brod and J. Combes, Phys. Rev. Lett. 117, 080502 (2016).

[33] M. Oszmaniec, R. Augusiak, C. Gogolin, J. Kołodyński, A. Acín, and M. Lewenstein, Phys. Rev. X 6, 041044 (2016).

[34] S. Bravyi, arXiv:0404180.

[35] B. C. Hall, Lie Groups, Lie Algebras, and Representations: An Elementary Introduction (Springer, New York, 2000).

[36] E. B. Dynkin, G. M. Seitz, and A. L. Onishchik, Selected Papers of E. B. Dynkin with Commentary (American Mathematical Society, Providence, 2000).

[37] G. M. Seitz, The Maximal Subgroups of Classical Algebraic Groups (American Mathematical Society, Providence, 1987), Vol. 365.

[38] See Supplemental Material http://link.aps.org/supplemental/ 10.1103/PhysRevLett.119.220502 for the proofs of technical results, which includes includes Refs. [39-44].

[39] J. Humphreys, Introduction to Lie Algebras and Representation Theory, Graduate Texts in Mathematics Series (Springer-Verlag GmbH, New York, 1972).
[40] J. J. Sakurai and J. Napolitano, Modern Quantum Mechanics (Addison-Wesley, Reading, MA, 2011).

[41] I. G. Macdonald, Symmetric Functions and Orthogonal Polynomials (American Mathematical Society, Providence, 1998).

[42] K. H. Hofmann and S. A. Morris, The Structure of Compact Groups: A Primer for the Student-A Handbook for the Expert (Walter de Gruyter, Berlin, 2013), Vol. 25.

[43] R. Zeier and Z. Zimborás, J. Math. Phys. 56, 081702 (2015).

[44] M. Oszmaniec and M. Kuś, J. Phys. A 45, 244034 (2012).

[45] F. G. S. L. Brandão, A. W. Harrow, and M. Horodecki, Phys. Rev. Lett. 116, 170502 (2016).

[46] S. Lloyd and S. L. Braunstein, Phys. Rev. Lett. 82, 1784 (1999).

[47] S. G. Schirmer, A. I. Solomon, and J. V. Leahy, J. Phys. A 35, 4125 (2002).

[48] F. Albertini and D. D'Alessandro, IEEE Trans. Autom. Control 48, 1399 (2003).

[49] M. Greiter, X.-G. Wen, and F. Wilczek, Phys. Rev. Lett. 66, 3205 (1991).

[50] F. Dolcini and A. Montorsi, Phys. Rev. B 88, 115115 (2013).

[51] D. J. Brod and A. M. Childs, Quantum Inf. Comput. 14, 901 (2014).

[52] S. Bravyi and D. Gosset, arXiv:1609.00735.

[53] M. A. Nielsen, M. R. Dowling, M. Gu, and A. C. Doherty, Science 311, 1133 (2006).

[54] A. W. Harrow, B. Recht, and I. L. Chuang, J. Math. Phys. 43, 4445 (2002). 